\title{
BIODEGRADABILIDAD DE LAS DIFERENTES FRACCIONES DE AGUA RESIDUAL PRODUCIDAS EN UNA TENERÍA
}

\section{BIODEGRADABILITY OF DIFFERENT FRACTIONS OF WASTEWATER PRODUCED IN A TANNERY}

\section{María Carolina Pire Sierra}

Ing. Químico. MSc. Profesor agregado. Programa de Ingeniería Agroindustrial. Decanato de Agronomía. Universidad Centroccidental Lisandro Alvarado. Barquisimeto, Venezuela.

carol_pire@yahoo.es

Karen Rodríguez Sargent

Ing. Civil. Departamento de Ingeniería Sanitaria y Ambiental. Facultad de Ingeniería.

Universidad del Zulia. Maracaibo, Venezuela.

karen_vrs@hotmail.com

Mary Fuenmayor Reyes

Estudiante Ing. Civil. Departamento de Ingeniería Sanitaria y Ambiental.

Facultad de Ingeniería. Universidad del Zulia. Maracaibo, Venezuela.

mary_eli20@hotmail.com

Yubislays Fuenmayor

Ing. Civil. Departamento de Ingeniería Sanitaria y Ambiental. Facultad de Ingeniería.

Universidad del Zulia. Maracaibo, Venezuela.

yubislays@hotmail.com

\section{Hervis Acevedo}

Ing. Civil. Departamento de Ingeniería Sanitaria y Ambiental. Facultad de Ingeniería.

Universidad del Zulia. Maracaibo, Venezuela.

hervis_20@hotmail.com

Sedolfo Carrasquero Ferrer

Ing. Químico. MSc. Becario Docente. Departamento de Ingeniería Sanitaria y Ambiental.

Facultad de Ingeniería. Universidad del Zulia. Maracaibo, Venezuela.

sedolfocarrasquero@gmail.com

Altamira Díaz Montiel

Ing. Civil. Ph.D. Profesor titular.Departamento de Ingeniería Sanitaria y Ambiental.

Facultad de Ingeniería. Universidad del Zulia. Maracaibo, Venezuela.

adiaz@fing.luz.edu.ve

Fecha de recepción: 22 de agosto de 2011

Fecha de aprobación: 19 de diciembre de 2011 


\title{
RESUMEN
}

Los efluentes de las tenerías están conformados por componentes orgánicos con diferentes niveles de biodegradabilidad, conocer su contenido biodegradable e inerte permite seleccionar los tratamientos de depuración más adecuados. El fraccionamiento de la materia orgánica, medida como demanda química de oxígeno (DOO), permitió cuantificar el contenido de DQO fácilmente biodegradable (DOOFB), DOO lentamente biodegradable (DOOLB), DOO no biodegradable soluble (DOONBs), y DOO no biodegradable particulada (DOONBp), contenidas en tres tipos de efluentes producidos en una tenería (mezcla pelambre-teñido, mezcla curtidoteñido y el efluente almacenado en una laguna). El objetivo fue seleccionar el efluente con mejores características de biodegradabilidad y así proponer los tratamientos de depuración más adecuados. Para tal fin, se utilizó un reactor por carga de $2 \mathrm{~L}$ de capacidad, alimentado con 70\% de efluente industrial y 30\% de biomasa. El sistema permaneció aireado durante el ciclo (24 h). Se midió la DOO total y soluble del agua residual cruda y licor mezcla, al inicio y al final del proceso. Se concluyó que el efluente con mayor contenido de DOO biodegradable fue el almacenado en la laguna con 57,4\%, conformado por 33,1\% de DOOFB y 24,3\% de DOOLB, mientras que los efluentes segregados P-T y C-T mostraron menor contenido de DOO biodegradable (35,8 y 34,0\%, respectivamente). Por lo tanto, el fraccionamiento de la DOO mostró que un tratamiento biológico sería más efectivo para tratar los efluentes almacenados en la laguna, porque podría remover hasta 57,4\% de DQO por vía biológica. Así mismo, los resultados mostraron que se requería de un postratamiento que removiera la parte coloidal de la fracción no biodegradable (29,0\% DOONBs y 13,6\% DOONBP).

Palabras clave: DQO biodegradable, DOO no biodegradable, materia orgánica, tenería.

\begin{abstract}
Tannery wastewater is composed by organic components with different biodegradability levels, so it is important to know its biodegradable and inert contents to select the most appropriate treatments. The organic matter fractionation, measured as chemical oxygen demand (COD), allowed to detect the contents of a readily biodegradable COD (rbCOD), slowly biodegradable COD (sbCOD), soluble inert COD (siCOD) and particulate inert COD (piCOD), contained in three types of tannery effluents produced (dyed-soak liquor mixture, dyed-tanning mixture, and pond-stored effluent). The goal of this research was to select the best effluent based on its biodegradability and propose the most appropriate purification treatments. For this investigation a $2 \mathrm{~L}-$ batch reactor of mixed liquor was used with $70 \%$ industrial effluent and 30\% biomass. The system remained aerated during the cycle (24 h). Total and soluble COD were measured for raw wastewater and mixed liquor at the beginning and end of the process. The effluent with the highest content of biodegradable COD was the mixture effluent stored in the pond with 57.4\%, formed by $33.1 \%$ rbCOD and $24.3 \%$ sbCOD, whereas the segregated mixtures showed a lesser biodegradable COD (35.8 and 34.0\%, respectively). Therefore, the COD fractionation showed that a biological treatment would be more effective to treat the effluent stored in the pond,
\end{abstract}


reaching 57.4\% biological removal of COD. Also, results showed a post-treatment is required to remove the colloidal part from a non-biodegradable fraction (29.0\% siCOD and $13.6 \%$ piCOD).

Keywords: organic matter, biodegradable COD, inert COD, tannery.

\section{INTRODUCCIÓN}

Las aguas residuales están constituidas por un amplio espectro de compuestos orgánicos con diversas propiedades físicas y diferentes niveles de biodegradabilidad. Para sistemas de lodos activados, es necesario conocer estas características en el agua residual, dado que informarán sobre el contenido del material soluble y particulado, biodegradable y no biodegradable [1].

Las características de biodegradabilidad son de vital importancia cuando se van a tratar aguas residuales que provienen de procesos industriales, particularmente de las tenerías o curtiembres, debido a que la DOO total que poseen esta agua, agrupa fracciones con marcadas diferencias en las tasas de biodegradabilidad, además de contener una importante fracción de DOO inerte o no biodegradable, características que han demostrado ser fundamentales para definir la extensión del tratamiento a aplicar [2] y [3].

La caracterización convencional de los efluentes de las tenerías que determina parámetros básicos como demanda química de oxígeno total $\left(\mathrm{DOO}_{\mathrm{t}}\right)$, demanda bioquímica de oxígeno $\left(\mathrm{DBO}_{5,20}\right)$, nitrógeno total Kjeldahl (NTK), nitrógeno amoniacal $\left(\mathrm{NH}_{4}{ }^{+}\right)$y sólidos suspendidos (SS), no proporciona información sobre las tasas de biodegradabilidad de sus componentes, por lo cual esos valores no pueden utilizarse exclusivamente para diseñar sistemas de tratamientos de aguas residuales industriales [2] y [3]. Por su parte, investigadores como Orhon et al. [2], Vidal et al. [4] y Lefebvre et al [5], señalaron que la $\mathrm{DBO}_{5,20}$ es considerada como un parámetro débil para efluentes de tenerías, debido a que este análisis sólo refleja una pequeña fracción del contenido orgánico presente en el efluente y además, su resultado es susceptible de estar distorsionado, debido a la presencia de sustancias inhibitorias en el agua residual como el cromo, característico de efluentes de tenerías. De allí que sea necesario hacer una caracterización más completa y profunda, conocida como fraccionamiento de la DOO, que permita conocer la composición del efluente en términos de las diferentes tasas de degradación de las fracciones de la DOO, y así determinar las fracciones biodegradables e inertes [6].

El fraccionamiento de la DOO divide este parámetro en dos grandes grupos: DOO biodegradable total (DOOBT) y DOO no biodegradable total o inerte (DOONBT). La fracción biodegradable se subdivide en DOO rápidamente biodegradable (DOORB), y DOO lentamente biodegradable (DOOLB). La primera es utilizada en el reactor biológico de forma inmediata, y se consume en los primeros minutos de haberse iniciado el tratamiento, provocando un incremento en el consumo de oxígeno por parte de los microorganismos que la usan para su síntesis celular. La fracción lentamente biodegradable debe ser primero absorbida sobre la 
estructura celular de los microorganismos que la hidrolizan a unidades químicas simples, utilizando enzimas extracelulares y finalmente, es metabolizada por ellos [1] y [7].

La fracción no biodegradable total se subdivide en DOO soluble inerte y DOO particulada inerte, que no podrán ser removidas por el sistema biológico. La primera de ellas junto a la pequeña cantidad de sólidos suspendidos o coloidales que se formarán en el reactor como producto de la actividad de los microorganismos, escaparán del sistema junto al efluente. Por su parte, la DOO particulada inerte se mezclará con la biomasa, sedimentando con ella, y es la responsable del aumento de la concentración de sólidos suspendidos en el licor mezcla [6] y [7]. Debido a la capacidad del lodo activado de flocular, todo el material sólido, biodegradable y no biodegradable, orgánico o inorgánico, se convertirá en sólido sedimentable. La masa de la DOO particulada inerte conformada por el residuo endógeno (paredes celulares), el material orgánico suspendido o sedimentable, se acumulará en el reactor, y sólo es retirado del mismo por medio de la purga. Es decir, la única forma de remover la fracción particulada de la DOO inerte, es por medio del control de la edad del lodo [1].

El sector productivo de las tenerías, se caracteriza por generar efluentes muy abundantes y con alto contenido de contaminantes puntuales como los sulfuros y metales pesados como el cromo, además de poseer altas concentraciones de materia orgánica, nitrógeno orgánico, nitrógeno amoniacal y sólidos suspendidos [8] y [9]. Diversos investigadores han recomendado segregar los efluentes producidos en las tenerías, para brindarle tratamiento por separado al agua residual resultante del proceso de pelambre que contiene sulfuros, de los provenientes del proceso de curtido que contienen cromo trivalente, y de esta manera, brindarle un tratamiento más efectivo a cada efluente, pudiendo incluso, recuperar o reciclar sustancias como el cromo [10] y [11].

Los tratamientos biológicos son más económicos y generan pocos subproductos en comparación con los tratamientos fisicoquímicos [12] Esta condición prevalece en muchos casos, para seleccionar los procesos biológicos como tratamientos para diversos efluentes; sin embargo, para poder definir el tipo de tratamiento más adecuado para los efluentes de la tenería, ya sean segregados o mezclados, es fundamental conocer el contenido de materia orgánica biodegradable e inerte de cada uno de ellos. Por lo tanto, en esta investigación se propuso realizar el fraccionamiento de la DOO de tres tipos de efluentes producidos en una tenería, como son las mezclas segregadas directamente del proceso productivo: pelambreteñido (P-T) y curtido-teñido (C-T), así como la mezcla de todos ellos pretratados y almacenados en una laguna (L) para seleccionar el efluente con mejores características de biodegradabilidad y con base en él, proponer un tratamiento que sea adecuado a las características del efluente de la tenería.

\section{MATERIALES Y MÉTODOS}

Los estudios se llevaron a cabo, utilizando aguas residuales de una tenería ubicada en el estado Lara (Venezuela), al Oeste del municipio Iribarren. La empresa trabajaba principalmente con pieles frescas de origen caprino (Capra hircus), y funcionaba de forma discontinua, por lo cual generaba tres tipos de efluentes durante la semana. El primero provenía del proceso de 
pelambre (P), el segundo del proceso de curtido $(C)$ y el tercero correspondía al proceso de teñido (T). Cada efluente se descargaba en un canal que desembocaba a una laguna que servía de reservorio donde ocurrían los procesos de homogenización y sedimentación.

Se utilizó la combinación P-T (50\% P, 30\% T y 20\% agua), y la mezcla C-T 160\% T, 20\% C y 20\% agua), debido a que en investigaciones previas, Pire et al. [13] y [14], usaron la fracción teñido como agente de dilución de las altas concentraciones de DOO y NTK contenidas en el pelambre, y el teñido también sirvió para disminuir la concentración de cromo presente en el efluente del proceso de curtido, hasta concentraciones inferiores a 180 que es la concentración a la cual Farabegoli et al. [15], observaron inhibición en el proceso biológico de nitrificación.

Para esta investigación, se fraccionó la materia orgánica de la mezcla P-T, la mezcla C-T y la mezcla de efluentes almacenados en la laguna, para conocer así cuál de ellas presentaba mejores condiciones de biodegradabilidad, y de esta manera, decidir el efluente de la tenería más adecuado para brindarle tratamiento biológico.

La caracterización convencional de cada efluente estudiado, se hizo determinando las concentraciones de DOO (SM 5220 C), demanda bioquímica de oxígeno, DBO 5,20 (SM 5210), nitrógeno total Kjeldahl, NTK (SM 4500-Norg B), nitrógeno amoniacal, $\mathrm{NH}_{4}{ }^{+}\left(\mathrm{SM} 4500-\mathrm{NH}_{3} \mathrm{D}\right)$, nitritos, $\mathrm{NO}_{2}^{-}\left(\mathrm{SM} 4110 \mathrm{~B}\right.$ ), nitratos, $\mathrm{NO}_{3}{ }^{-}(\mathrm{SM} 4110 \mathrm{~B}$ ), sólidos suspendidos totales, SST (SM 2540 D), sólidos suspendidos volátiles, SSV (SM 2540 E), cromo total (3111 B) y pH (SM 4500 HB), siguiendo los procedimientos establecidos en el método estándar [16].

El fraccionamiento se realizó en un reactor por carga cilíndrico de 14,5 cm de diámetro y $26 \mathrm{~cm}$ de alto, con un volumen total del dispositivo de $4 \mathrm{~L}$. El volumen de trabajo fue de $2 \mathrm{~L}$, del cual un 30\% correspondió a lodo activado (previamente aclimatado), y el resto a agua residual (Figura 1).

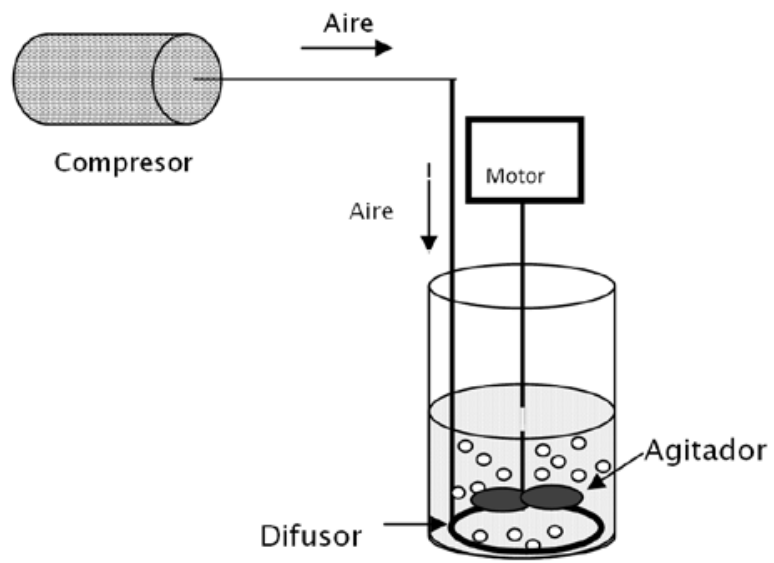

Figura 1. Esquema del reactor por carga para el fraccionamiento de la DOO 
En el interior del reactor, se colocó un difusor por donde se inyectó aire proveniente de un

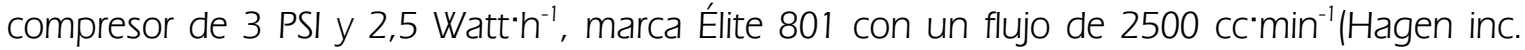
China). La agitación se hizo mecánica, con un aspa sumergida en el reactor, y movida por un motor monofásico SE Motors modelo SE24 de 1/20 HP, 115 v, $60 \mathrm{~Hz}$ y 2,1 A. La temperatura del fraccionamiento osciló entre 28 y $30^{\circ} \mathrm{C}$ durante todos los experimentos.

El procedimiento utilizado para fraccionar la materia orgánica fue el señalado por Park et al. [7], que consistió en determinar la DOO biodegradable y la no biodegradable mediante un reactor por carga que funcionó en condiciones aeróbicas durante 24 horas. Se estableció este tiempo, debido a que se asumió que en ese período, todo el material orgánico particulado biodegradable había sido hidrolizado y el proceso de oxidación biológica se había completado. Para la determinación de la DOO biodegradable total (DOOBT), se midió la DOO total y soluble (filtrada por membrana de 0,45 $\mu \mathrm{m}$ ), tanto del agua residual cruda como del licor mezcla, al inicio del tratamiento (hora 0). Al cabo de 24 horas, se midió nuevamente la DOO soluble del agua residual (DOONBS).

La determinación de la DQO fácilmente biodegradable (DOOFB), se hizo midiendo la DOO del agua residual previamente sometida a un proceso de coagulación-floculación con $\mathrm{ZnSO}_{4}$ (100mg L-1) a $\mathrm{pH} 10,5$, tanto del agua residual cruda, como del efluente, luego de permanecer 24 horas en el reactor por carga, como manera de obtener la DOO verdaderamente soluble en cada uno de los efluentes estudiados. Conociendo la DOOBT y la DOOFB, por diferencia se obtuvo la DOO lentamente biodegradable (DOOLB). Por su parte, la DQO no biodegradable particulada (DOONBp), también se obtuvo por diferencia entre la DOO total, DOOBT y DOONBs [7]. Este procedimiento se aplicó exitosamente en efluentes industriales provenientes de la industria avícola [17].

La caracterización de los tres efluentes estudiados en esta investigación se realizó mensualmente tomando las aguas residuales en la empresa el día que se producían o directamente de la laguna utilizando envases plásticos de 30 L. La frecuencia de toma de muestras en el reactor por carga para el fraccionamiento de la DOO fue interdiaria por un período mínimo de tres semanas, para un total de 9 muestras para cada tipo de efluente estudiado. Todos los parámetros medidos en la caracterización convencional, así como en el fraccionamiento fueron analizados por duplicado.

Diseño de la investigación. Se hizo la caracterización convencional mediante estadística descriptiva, reportando la tendencia central y su dispersión (DE: desviación estándar o C.V.: coeficiente de variación). Por su parte, el experimento del fraccionamiento de la DOO se condujo con un diseño completamente al azar, con tres tratamientos (P-T, C-T, L), cuatro variables (DOOFB, DOOLB, DOONBS, DOONBP), y nueve repeticiones en el tiempo. Los resultados se compararon, utilizando el análisis de mediciones repetidas con el programa estadístico Statistix versión 8.0 para un grado de confianza del 5\%. 


\section{RESULTADOS Y ANÁLISIS}

\subsection{CARACTERIZACIÓN CONVENCIONAL}

Las características de las aguas residuales producidas en las tenerías, son muy variables debido a las particularidades de los procesos productivos de cada empresa, tamaño de la tenería, tipos de reactivos químicos usados en procesos específicos, cantidad de agua usada y tipo de producto final [3]. La caracterización de los efluentes de la empresa dedicada al curtido de cuero, es un requisito fundamental debido a la fuerte variabilidad en la concentración de sus componentes. Por lo tanto, para este estudio se hizo la caracterización fisicoquímica del agua residual de la tenería para las mezclas P-T, C-T y L (Tabla 1).

Tabla 1. Caracterización convencional de los efluentes producidos en la tenería

\begin{tabular}{|c|c|c|c|}
\hline Parámetro & $\begin{array}{l}\text { Efluente Laguna } \\
\text { (media } \pm \text { DE) }\end{array}$ & $\begin{array}{c}\text { Mezcla } \\
\text { Pelambre-Teñido } \\
\text { (media } \pm \text { DE) } \\
\end{array}$ & $\begin{array}{c}\text { Mezcla } \\
\text { Curtido-Teñido } \\
\text { (media } \pm \text { DE) } \\
\end{array}$ \\
\hline $\mathrm{pH}$ & $8,1 \pm 0,4$ & $8,0 \pm 0,8$ & $3,5^{\star} \pm 0,1$ \\
\hline SST $\left(m g \mathrm{~L}^{-1}\right)$ & $2.310,0 \pm 415,0$ & $9.445,0 \pm 4.665,0$ & $3.475,0 \pm 1.164,4$ \\
\hline $\mathrm{SSV}\left(\mathrm{mg} \cdot \mathrm{L}^{-1}\right)$ & $960 \pm 90,0$ & $7.380,0 \pm 4.170$ & $1.120,0 \pm 324,4$ \\
\hline DQO total $\left(\mathrm{mg}^{\prime} \mathrm{L}^{-1}\right)$ & $2.510,2 \pm 423,7$ & $38.191,1 \pm 7.117,8$ & $6.421,1 \pm 234,8$ \\
\hline $\mathrm{DBO}_{5,20}\left(\mathrm{mg}^{\left.\cdot \mathrm{L}^{-1}\right)}\right.$ & $1.053,1 \pm 134,1$ & $11.760,0 \pm 347,6$ & $1.387 .5 \pm 53,0$ \\
\hline $\mathrm{DBO}_{5,20} / \mathrm{DOO}_{\text {total }}(\%)$ & 42,0 & 30,8 & 21,6 \\
\hline NTK $\left(\mathrm{mg}^{\circ} \mathrm{L}^{-1}\right)$ & $147,0 \pm 19,6$ & $1.729,0 \pm 682,5$ & $185,2 \pm 26,4$ \\
\hline $\mathrm{NH}_{4}^{+}\left(\mathrm{mg} \mathrm{L}^{-1}\right)$ & $75,3 \pm 15,2$ & $108,5 \pm 7,0$ & $138,0 \pm 17,3$ \\
\hline $\mathrm{NO}_{2}^{-}\left(\mathrm{mg}^{\cdot} \mathrm{L}^{-1}\right)$ & $1,3 \pm 0,9$ & ND & ND \\
\hline $\mathrm{NO}_{3}^{-}\left(\mathrm{mg}^{\cdot} \mathrm{L}^{-1}\right)$ & $2,7 \pm 3,0$ & ND & ND \\
\hline Cromo total $\left(\mathrm{mg} \mathrm{L}^{-1}\right)$ & $4,2 \pm 0,5$ & $13,7 \pm 3,5$ & $160,6 \pm 81,3$ \\
\hline
\end{tabular}

El efluente proveniente de la mezcla P-T, se caracterizó por contener una DOO total de $38.191,1 \mathrm{mg} \cdot \mathrm{L}^{-1}$ y una concentración de NTK de $1.729,0 \mathrm{mg}^{\circ} \mathrm{L}^{-1}$; estas elevadas concentraciones se debieron a que el efluente generado durante el proceso de pelambre era rico en proteínas y grasas que se removían de las pieles de los animales durante la etapa de limpieza, y por los elevados niveles de nitrógeno que provenían de las sales de amonio que se añadieron durante el pelambre y de los compuestos nitrogenados que contenían los colorantes usados en las pieles durante la etapa de teñido [8].

Por su parte, el efluente formado por la mezcla C-T tuvo una DOO total de $6.421,1 \mathrm{mg}^{-\mathrm{L}^{-1}}$, un contenido de NTK de 185,2 mg'L-1 y una concentración de cromo total de 160,6 $\mathrm{mg}^{\cdot \mathrm{L}^{-1}}$. Las concentraciones de los contaminantes en este efluente, con excepción del $\mathrm{Cr}$, fueron inferiores a las conseguidas para la mezcla P-T. Sin embargo, siguió correspondiendo a un efluente fuerte (DOO y NTK superiores a 2.000 y $100 \mathrm{mg}^{\circ} \mathrm{L}^{-1}$, respectivamente), [2] y [3]. De igual manera, el nitrógeno provino de los colorantes añadidos durante el teñido y la elevada concentración de 
cromo fue producto de la adición de sales de este metal durante el curtido de las pieles para prevenir su pudrición. El pH ácido se debió a la adición de ácido sulfúrico utilizado para mantener el cromo en solución acuosa [15].

Por último, el efluente almacenado en la laguna fue el que presentó menores concentraciones de contaminantes entre los tres efluentes caracterizados, con una DOO de $2.510,2 \mathrm{mg}^{\circ} \mathrm{L}^{-1}$, NTK de $185,2 \mathrm{mg}^{-1} \mathrm{~L}^{-1}$ y una concentración de cromo total de $4,2 \mathrm{mg} \mathrm{L}^{-1}$, lo cual indicó que el almacenamiento del agua residual en la laguna, sirvió como un pretratamiento donde ocurrió el proceso de homogenización y sedimentación por gravedad, así como reacciones de degradación que contribuyeron a remover parte de la DOO total y a estabilizar el pH en 8 unidades. Bajo estas condiciones de $\mathrm{pH}$, el cromo no es soluble y por lo tanto, se precipitó junto a otros componentes sedimentables, haciendo que disminuyeran sus concentraciones en la fracción líquida del agua residual [15].

El contenido de sólidos suspendidos fue elevado en todos los efluentes estudiados, y coincide con lo señalado por Ganesh et al. [9] y Lefebvre et al. [11], quienes afirmaron que es una característica propia de los efluentes de tenerías que oscilen entre 5.300,0 y 10.700,0 mgSS $\mathrm{L}^{-1}$. También es importante resaltar que los efluentes empleados para las mezclas P-T y C-T, no reportaron presencia de nitritos ni nitratos (límite de detección $1 \mathrm{mg}^{*} \mathrm{~L}^{-1}$ ), indicando que por haberlos tomado tan pronto se produjeron en la tenería (efluentes frescos), no tuvieron alguna transformación biológica, mientras que en el efluente almacenado en la laguna, sí se detectó la presencia de estos iones, indicando que al menos el proceso de nitrificación había ocurrido parcialmente.

Los resultados de la relación de biodegradabilidad ( $\mathrm{DBO}_{5,20} / \mathrm{DQO}$ total), con base en los criterios establecidos por Ahn et al. [18] e INESCOP [19], mostraron que la mezcla C-T debería ser catalogada como no biodegradable (DBO/DOO < 0,3), mientras que la mezcla P-T y L serían poco biodegradables (DBO/DOO entre 0,3 y 0,7). Según estos resultados, no sería viable ni recomendable aplicar un sistema de tratamiento biológico en las aguas residuales de tenerías. Sin embargo, investigadores como Vidal et al. [4] y Lefebvre et al. [5], lograron aplicar con éxito, tratamientos biológicos a efluentes de tenerías con relaciones DBO/DOO de 0,3, lo cual indica que la relación de biodegradabilidad mencionada no es adecuada para estos efluentes industriales. Una de las razones por las cuales la relación $\mathrm{DBO}_{5,20} / \mathrm{DOO}_{\text {total }}$ no es precisa, es porque la $\mathrm{DBO}_{5,20}$ no es un buen parámetro de referencia cuando se emplean aguas residuales de tenerías, debido a que la compleja matriz de compuestos tóxicos y no tóxicos que la conforman, son inhibitorios para la prueba de $\mathrm{DBO}_{5,20}$ [4] y [5].

La caracterización convencional de cada efluente de la tenería, determinó que parámetros comunes como SST, DOO, DBO ${ }_{5,20}$, y nitrógeno total (NTK $+\mathrm{NH}_{4}{ }^{+}+\mathrm{NO}_{2}{ }^{-}+\mathrm{NO}_{3}{ }^{-}$), no cumplieron con los límites de descarga establecidos en la normativa venezolana vigente [20]. Por esta razón, es necesario que dichos efluentes sean sometidos a algún tipo de tratamiento físico, químico y/o biológico antes de ser descargados a cuerpos de agua. Sin embargo, para poder seleccionar el tratamiento más conveniente para los efluentes producidos en las tenerías, 
se hace necesario conocer con más detalle, sus características en cuanto a la biodegradabilidad de parámetros importantes como es la DOO [3].

\subsection{FRACCIONAMIENTO DE LA DEMANDA OUÍMICA DE OXÍGENO}

El fraccionamiento de la DOO fue una herramienta útil en la determinación de la biodegradabilidad de las aguas residuales, debido a que midió el contenido de materia orgánica biodegradable y la no biodegradable, así como también clasificó la DOO de los efluentes mezclados de la tenería, según su velocidad de degradación y sus características solubles o particuladas $[1,2,7]$. En base a la información generada por el fraccionamiento de los efluentes mezclados de la tenería se pudo seleccionar el efluente con mejores características de tratabilidad para luego definir el tratamiento de depuración más conveniente [3,21].

La relación convencional de biodegradabilidad, que relaciona la $\mathrm{DBO}_{5,20} / \mathrm{DQO}_{\text {total }}$ (Tabla 1) es inferior al contenido de material biodegradable (DOOBT) obtenido mediante la prueba del fraccionamiento de la DOO (Tabla 2), especialmente para el efluente almacenado en la laguna y para la mezcla C-T. Estos resultados reforzaron que la $\mathrm{DBO}_{5,20}$ tampoco fue un buen parámetro de referencia cuando se emplearon los efluentes de tenerías estudiados en esta investigación, coincidiendo con lo señalado por Vidal et al. [4] y Lefebvre et al. [5].

Tabla 2. Fraccionamiento de la materia orgánica para los efluentes en estudio producidos en la tenería. En paréntesis el C.V.

\begin{tabular}{|c|c|c|c|}
\hline Fraccionamiento DQO & Efluente Laguna & $\begin{array}{c}\text { Mezcla } \\
\text { Pelambre-Teñido }\end{array}$ & $\begin{array}{c}\text { Mezcla } \\
\text { Curtido-Teñido }\end{array}$ \\
\hline \multicolumn{5}{|c|}{ GENERAL } \\
\hline DOOBT (\%) & $57(17,4)$ & $35,8(19,0)$ & $34,0(14,7)$ \\
\hline DOONBT (\%) & $42,6(23,5)$ & $64,2(10,6)$ & $66,0(7,6)$ \\
\hline \multicolumn{5}{|c|}{ DETALLADO* } \\
\hline DOOFB (\%) & $33,1 \mathrm{a}$ & $19,1 \mathrm{~b}$ & $17,9 \mathrm{~b}$ \\
\hline DQOLB (\%) & $24,3 \mathrm{a}$ & $16,7 \mathrm{~b}$ & $16,1 \mathrm{~b}$ \\
\hline DOONBs (\%) & $29,0 \mathrm{~b}$ & $26,0 \mathrm{~b}$ & $40,4 \mathrm{a}$ \\
\hline DOONBP (\%) & $13,6 \mathrm{c}$ & $38,2 \mathrm{a}$ & $25,6 \mathrm{~b}$ \\
\hline
\end{tabular}

*Media seguida por letras distintas en cada fila, indica diferencias significativas según la prueba de Tukey $(P \leq 0,05)$

La caracterización y el fraccionamiento del agua residual proveniente de la laguna de almacenamiento, mostraron que la DOO total fue de $2.510,2 \mathrm{mg}^{\cdot \mathrm{L}^{-1}}$, con una fracción biodegradable de 57,4\% y una fracción no biodegradable de 42,6\%. Por su parte, las mezclas de pelambre-teñido, como de curtido-teñido presentaron concentraciones muy diferentes de DOO total, siendo $38.191,1 \mathrm{mg} \mathrm{L}^{-1}$ para la primera y $6.421,1 \mathrm{mg} \mathrm{L}^{-1}$ para la segunda (Tabla 1). Sin embargo, el fraccionamiento mostró que tuvieron un comportamiento similar entre sí, respecto de las fracciones de DOO biodegradable con 35,8\% para la mezcla P-T y 34\% para la mezcla C-T, mientras que el resto de la DOO total correspondió a la fracción inerte 164,3 y 
66\%, respectivamente). Estos resultados mostraron la diferencia que existe entre la DOO total y la DOO biodegradable. De allí la importancia de conocer las fracciones de la DOO para hacer un diseño más adecuado de los sistemas de tratamientos biológicos, puesto que el diseño se basaría en el contenido de la materia orgánica biodegradable en lugar de la materia orgánica total [17] y [22].

Los resultados obtenidos del fraccionamiento de la materia orgánica de los efluentes de tenerías estudiados en esta investigación, se encontraron dentro del rango señalado en la literatura para estos efluentes industriales. Al respecto, Insel et al. [23], encontraron que la fracción biodegradable del efluente sedimentado de una tenería fue de $81 \%$, mientras que Karahan et al. [3] establecieron que sólo el 34,9\% del efluente de una tenería era biodegradable. Estos resultados muestran la variabilidad en la biodegradabilidad que presentan los efluentes de las tenerías, y atribuye a la diferencia de compuestos químicos adicionados durante cada proceso, tipo de pieles procesadas, así como también a la existencia o no de un pretratamiento como la sedimentación [2]. Al respecto, el efluente almacenado en la laguna, mostró mejores características de biodegradabilidad con un porcentaje de DOOBT $(57,4 \%)$ superior que al de las mezclas P-T y C-T (35,8 y 34,0\%, respectivamente), demostrando así, que el proceso de sedimentación y las reacciones de degradación asociadas que ocurrieron en la laguna, contribuyeron a remover parte de la DOO total, mientras que esto no ocurrió en los efluentes recién tomados del proceso productivo (P-T y C-T).

La DOO fácilmente biodegradable (DOOFB), para el efluente almacenado en la laguna, fue de $33,1 \%$, superior a la DOO lentamente biodegradable (24,3\%), obtenida para ese mismo efluente. La DOOFB del efluente almacenado en la laguna, fue superior a lo reportado por otros investigadores que también utilizaron aguas residuales de tenerías, cuyo rango estuvo entre 7,5 y 19\% [2], [3] y [23]. Sin embargo, se comportó de manera similar a la DOOFB reportada por Palmero et al. [17] quienes al realizar el fraccionamiento de la DOO a un efluente avícola, consiguieron que la DOOFB (58\%), fuera superior a la DOOLB (31\%), para una DOO total de $1.840,4 \mathrm{mg}^{\circ} \mathrm{L}^{-1}$.

Por su parte, la DOOFB de las mezclas P-T fue de 19,1\% y para la mezcla C-T fue de 17,9\%, siendo ligeramente superiores a sus correspondientes DOOLB (16,7 y 16,1\%, respectivamente), lo cual representa para los dos tipos de efluentes, cerca del 50\% de la DOOBT. Los resultados de la DOO fácilmente biodegradable de las mezclas P-T y C-T, estuvieron dentro del rango obtenido por investigadores como Insel et al. [23] y Hermida-Veret et al. [21], quienes obtuvieron fracciones de DOOFB entre 15 y $20 \%$ de la DOO total en aguas residuales de tenerías.

La importancia de conocer el contenido de DOOFB en las aguas residuales industriales, es que este tipo de materia orgánica es la que requieren los microorganismos en los procesos de remoción de nitrógeno por vía biológica. Durante la etapa de desnitrificación, se requiere aproximadamente $7 \mathrm{~g}$ de DOOFB para remover $1 \mathrm{~g}$ de $\mathrm{NO}_{3}{ }^{-}$[24]. Por lo tanto, es mejor utilizar el efluente que posea mayor contenido de DOOBT para realizarle el tratamiento biológico, en particular aquél que posea mayor fracción fácilmente biodegradable, debido a que la DQO está 
disponible de manera inmediata para los microorganismos, mientras que la fracción lentamente biodegradable, primero debe ser hidrolizada por los microorganismos, y requiere de más tiempo para estar disponible [1] y [7].

El fraccionamiento de la DOO mostró que la DOOLB para el efluente de la laguna fue de 24,3\%, similar a la obtenida por Karahan et al. [3], para efluentes de tenerías que reportaron 27,6\% de DOOLB. Sin embargo, otros investigadores consiguieron que la fracción lentamente biodegradable en efluentes de tenerías correspondió al 60\% de la DOO [22] y [23]. Por otra parte, para las mezclas P-T y C-T, la DOOLB estadísticamente fue similar entre sí 116,7 y 16,1\%, respectivamente), pero menor a la reportada en la literatura.

La variación en los porcentajes obtenidos de DOOLB, resalta la importancia de conocer las fracciones de la DOO, particularmente la DOOLB porque está relacionada con la duración del tratamiento biológico [2]; por lo tanto, esta fracción permitiría estimar el tiempo de residencia hidráulico para cada tipo de efluente estudiado. En este caso en particular, la duración del tratamiento biológico sería inferior, si se usaran reactores biológicos alimentados con cualquiera de las mezclas (P-T O C-T), debido a que la fracción que debe ser hidrolizada por los microorganismos, es menor para las mezclas que para el efluente de la laguna (DOOLB mezcla $<$ DOOLB laguna). Sin embargo, no es el único criterio que se aplicaría en la selección del efluente por tratar, debido a que C-T y P-T tuvieron el menor contenido de DOOBT.

El contenido de DOO no biodegradable (DOONBT), en el efluente almacenado en la laguna, fue de 42,6\%, constituido en su mayoría por la DOO soluble inerte (29\%), mientras que el $13,6 \%$ restante corresponde a la fracción particulada inerte. Por su parte, la DOONBT de mezcla P-T fue de $64,2 \%$, conformada por $26,0 \%$ de DOONBs y $38,2 \%$ de DOONBp, mientras que para la mezcla C-T la DOONBT fue de 66\%, de la cual el 40,4\% es soluble inerte (DOONBs), y el 25,6\% restante correspondió a la forma particulada inerte. Así se evidencia que las mezclas P-T y $\mathrm{C}-\mathrm{T}$ presentaron los mayores contenidos de material inerte o no biodegradable.

La fracción soluble inerte fue estadísticamente similar para el efluente almacenado en la laguna y para la mezcla P-T ( $>0,05)$, y la mezcla C-T fue la que tuvo el mayor porcentaje de DOONBs $(p \leq 0,05)$. El contenido de material soluble inerte es el responsable de las bajas eficiencias de remoción reportadas por algunos investigadores cuando le aplicaron tratamiento biológico a efluentes de tenerías [13] y [14], debido a que esta fracción de la DOO no puede ser removida por el sistema biológico. Sólo tratamientos fisicoquímicos como adsorción, coagulaciónfloculación y entrampamientos físicos, serían capaces de remover los componentes coloidales de esta fracción [3].

Además, la fracción particulada inerte del efluente almacenado en la laguna, estadísticamente fue inferior al obtenido para la mezcla C-T y éste a su vez, inferior al de la mezcla P-T ( $\leq \leq 0,05)$. La importancia de conocer la DOONBp radicó en que es la fracción capaz de entrelazarse con la biomasa de un sistema biológico y separarse del agua residual por sedimentación [1]. Sin embargo, sólo sería retirada del sistema por medio del control de la edad del lodo (purga). Por lo tanto, para la mezcla P-T se recomendaría emplear una edad de lodo más baja que para la 
mezcla C-T y a su vez más baja que para el efluente almacenado en la laguna. Esto implicaría realizar volúmenes de purgas más altas a las mezclas segregadas que al efluente tomado de la laguna y por lo tanto, evitar la acumulación de esta DOO particulada en el sistema biológico. No obstante, la selección de la edad del lodo dependería no sólo del contenido de la DOONBp, sino también del objetivo del tratamiento biológico [1], [25] y [26].

El contenido de DOONBp del efluente de la laguna fue de 13,6\%, similar al reportado por Orhon y col. [2] e Insel y col. [23], quienes consiguieron 11,0 y 11,5\% de esta fracción, mientras que el porcentaje reportado para las mezclas P-T y C-T fue significativamente superior (38,2 y 25,6\%, respectivamente). Todos Ios resultados estuvieron dentro del amplio rango de variación de la DOONBp reportado por Karahan y col. [3] e Insel y col. [23], que osciló entre 11,0 a 52,2\% de la DQO total.

El elevado porcentaje de la fracción particulada inerte de las mezcla P-T y C-T se atribuyó a la cantidad de partículas suspendidas que poseían los efluentes segregados que fueron tomados justo luego de ser producidos. Para la mezcla P-T el contenido de SST fue en promedio de $9.445,0 \mathrm{mg} \mathrm{L}^{-1}$, mientras que los SSV promedio fueron de $7.380,0 \mathrm{mg}^{\circ} \mathrm{L}^{-1}$. Para la mezcla C-T los SST y SSV fueron de $3.475,0$ y $1.120,0 \mathrm{mg}^{\circ} \mathrm{L}^{-1}$ en promedio, respectivamente (Tabla 1). HermidaVeret y col. [21], señalaron que la sedimentación química fue capaz de remover parte de esta DOO particulada inerte, lo cual mejoró la calidad del agua residual antes de alimentarse de un sistema de tratamiento biológico y sin embargo, el efluente aún contenía un alto porcentaje de DOONBp. Al respecto, Orhon y col. [2] obtuvieron un efluente con contenido particulado casi nulo, luego de aplicarle una sedimentación química al efluente de la tenería.

La comparación del contenido de materia orgánica biodegradable entre los tres tipos de efluentes estudiados en esta investigación, demostró que el agua residual proveniente de la laguna de almacenamiento presentó mejores características de biodegradabilidad y por lo tanto, es el más adecuado para brindarle tratamiento, preferiblemente biológico, debido a que se lograrían remociones cercanas al 60\% (57,4\% de DOOBT). Sin embargo, un importante porcentaje de DOONBT quedaría remanente en el efluente tratado, por lo cual se debería incorporar un postratamiento fisicoquímico para remover la fracción coloidal de la DOONBT.

Finalmente, los resultados del fraccionamiento de la DOO también mostraron que el efluente de la laguna, al poseer el menor contenido de DOONBp, permitiría que se utilizaran edades de lodo de intermedias a altas (10-20 días), durante el tratamiento biológico, lo cual favorecería los procesos de nitrificación y desnitrificación y por lo tanto, la eficiencia del sistema biológico para la remoción de nutrientes [25] y [26].

\section{CONCLUSIONES}

La caracterización de los efluentes de la tenería, permitió seleccionar el efluente almacenado en la laguna, como el más adecuado para aplicarle tratamiento de depuración debido a que 
presentó las menores concentraciones de contaminantes y el mayor contenido de material biodegradable $(57,4 \%)$.

La DOOBT del efluente almacenado en la laguna, estuvo constituida por 33,1\% de DOOFB y $24,3 \%$ de DOOLB, mientras que la DOONBT quedó conformada por 29\% de DOONBs y $13,6 \%$ de DOONBP.

El sistema de tratamiento recomendado para el efluente almacenado en la laguna, debería estar conformado por un sistema biológico para remover al menos, 57,4\% de su DOO total y un postratamiento fisicoquímico para remover la parte coloidal de la fracción no biodegradable (42,6\%).

La sedimentación y las reacciones de degradación ocurridas en el efluente almacenado en la laguna, favorecieron la biodegrabilidad de esta agua residual, incrementando el contenido de DOO fácilmente biodegradable a 33,1\%, mientras que para las mezclas P-T y C-T, tomadas justo luego de ser producidas, fue de 19,1 y 17,9\%.

\section{REFERENCIAS BIBLIOGRÁFICAS}

[1] Ekama G. and Wentzel M. (2008). Biological Wastewater Treatment: Principles, Modelling and Design. Published by IWA Publishing, London, UK.

[2] Orhon D., Genceli E., and Ubay E. (1999). Characterization and modelling of activated sludge for tannery wastewater. In: Water Environment Research Vol. 71 (1), pp. 50-63.

[3] Karahan Ö., Dogruel S., Dulekgurgen E., and Orhon D. (2008). COD fractionation of tannery wastewaters - Particle size distribution, biodegradability and modeling. In: Water Research 42, 1083-1092.

[4] Vidal G., Nieto J., Cooman K., Gajardo M., and Bornhardt C. (2004). Unhairing effluents treated by an activated sludge system. In: Journal of Hazardous Materials B1 12, pp. 143149.

[5] Lefebvre O., Vasudevan N., Torrijos M., Thanasekaran K., and Moletta R. (2005). Halophilic biological treatment of tannery soak liquor in a sequencing batch reactor. In: Water Research. Vol 39, pp. 1471-1480.

[6] Orhon D., Artan N., and Ates E. (1994). A description of three methods for the determination of the initial inert particulate chemical oxygen demand of wastewater. In: Journal of Chemical Technology and Biotechnology. Vol 61, No 1, pp. 73-80. 
[7] Park J., Wang J., and Novotny G. (1997). Wastewater characterization for evaluation of biological phosphorus removal. In: Research Report. Vol 174, pp. 1-26.

[8] Stoop M.L.M. (2003). Water management of production systems optimised by environmentally oriented integral chain management: case study of leather manufacturing in developing countries. In: Technovation. Vol 23, pp. 265-278.

[9] Ganesh R., Balaji G., and Ramanujam R. (2006). Biodegradation of tannery wastewater using sequencing batch reactor - Respirometric assessment. In: Bioresource Technology. Vol 97. No 15, pp.1815-1821.

[10] Integrated Pollution Prevention and Control of the European Commission (IPPC). (2003). Reference Document on Best Available Techniques for the Tanning of Hides and Skins. European Commission.

[1 1] Lefebvre O., Vasudevan N., Torrijos M., Thanasekaran K., and Moletta R. (2006). Anaerobic digestión of tannery soak liquor with an aerobic post-treatment. In: Water Research. Vol 40, pp. 1492-1500.

[12] López-López A., Barrera-Fraire J., y Vallejo-Rodríguez R. (2008). Estudio comparativo entre un proceso fisicoquímico y uno biológico para tratar agua residual de rastro. In: Interciencia. Vol. 33 (7), pp. 490-496.

[13] Pire M.C., Palmero J., Araujo I., y Díaz A. (2010a). Tratabilidad del efluente de una tenería usando un reactor por carga secuencial. En: Revista Científica, FCV-LUZ. Vol XX, No 3, pp. 284-292.

[14] Pire M.C., Palmero J., Araujo I., y Díaz A. (2010b). Tratabilidad del efluente de una tenería con presencia de cromo usando un reactor por carga secuencial. En: Revista Científica, FCV-LUZ. Vol. XX (4), pp. 390-398.

[15] Farabegoli G., Caruccí A., Majone M. and Rolle E. (2004). Biological treatment of tannery wastewater in the presence of chromium. In: Journal of Environmental Management. Vol 71, pp. 345-349.

[16] American Public Health Association (APHA-AWWA-WEF). (1998). Standard Methods for the Examination of Water and Wastewater. $20^{\text {th }}$ edition. American Public Health Association, Washintong.

[17] Palmero J., Pire M.C., Hernández J.L., López F.L., Rincón N.C., y Díaz A.R. (2009). Fraccionamiento de la materia orgánica de un agua residual de la industria avícola para la remoción biológica de nutrientes. En: Boletin del Centro de Investigaciones Biológicas. Vol. 43 (2), pp. 211-224. 
[18] Ahn D.H., Chang W.S., and Yoon T.I. (1999). Dyestuff wastewater treatment using chemical oxidation, physical adsorption and fixed bed biofilm process. In: Process Biochem. Vol. 34, pp 429-439.

[19] INESCOP. Centro de Innovación y Tecnología (2008). Aplicación de bioensayos respirométricos en aguas de tenerías. Departamento del Medio Ambiente de INESCOP. Alicante, España.

[20] Ministerio del Ambiente de Recursos Naturales Renovables (1995). Normas para la clasificación y el control de la calidad de los cuerpos de agua y vertidos o efluentes líquidos. Decreto 883. Gaceta Oficial 5021. Venezuela.

[21] Hermida-Veret S., Galisteo M., y Viñas M. (2000). Evaluación respirométrica de la biodegradabilidad aeróbica de un efluente de curtiembre. XXVII Congresso Interamericano de Engenharia Sanitária e Ambiental. Trabajo en extenso l-184. pp 1-7. Porto Alegre, Brasil.

[22] Ubay E., Sözen S., Orhon D., and Henze M. (1998). Respirometric analysis of activated sludge behavior - I. Assessment of the readily biodegradable substrate. In: Water Research. vol. 32 (2), pp. 461-475

[23] Insel H., Görgün E., Artan N., and Orhon D. (2009). Model based optimization of nitrogen removal in a full scale activated sludge plant. In: Environmental Engineering Science. Vol. 26 (3), pp. 471-479.

[24] Insel G. (2007). Effects of design and aeration control parameters on simultaneous nitrification and denitrification (SNdN) performance for activated sludge process. In: Environmental engineering Science. Vol. 24 (5), pp. 675-686.

[25] Palma M., y Manga J. (2005). Simulación de un sistema de fangos activados en discontinuo (SBR) para el tratamiento de aguas residuales con altos contenidos de nitrógeno. En: Ingeniería y desarrollo. Vol. 2 (18), pp. 61-71.

[26] González M., y Saldarriaga J. (2008). Remoción biológica de materia orgánica, nitrógeno y fósforo en un sistema tipo anaerobio. En: Revista EIA. Vol. 10, pp. 45-53. 
